\title{
Brock malignancy risk calculator for pulmonary nodules: validation outside a lung cancer screening population
}

\author{
Kaman Chung, ${ }^{1}$ Onno M Mets, ${ }^{2}$ Paul K Gerke, ${ }_{1}^{1}$ Colin Jacobs, ${ }^{1}$ \\ Annemarie M den Harder, ${ }^{2}$ Ernst T Scholten, ${ }^{1}$ Mathias Prokop, ${ }^{1}$ Pim A de Jong, ${ }^{2}$ \\ Bram van Ginneken, ${ }^{1}$ Cornelia M Schaefer-Prokop ${ }^{1,3}$
}

${ }^{1}$ Department of Radiology and Nuclear Medicine, Radboud University Medical Center, Nijmegen, The Netherlands ${ }^{2}$ Department of Radiology, University Medical Center Utrecht, Utrecht, The Netherlands

${ }^{3}$ Department of Radiology, Meander Medical Center, Amersfoort, The Netherlands

Correspondence to Dr Kaman Chung, Department of Radiology and Nuclear Medicine, Radboud University Medical Center, Nijmegen, 6525 GA, The Netherlands; kaman.chung10@gmail.com

Received 4 December 2017 Revised 18 April 2018 Accepted 30 April 2018 Published Online First 18 May 2018
Check for updates

To cite: Chung K, Mets OM, Gerke PK, et al. Thorax 2018:73:857-863.

\section{ABSTRACT}

Objective To assess the performance of the Brock malignancy risk model for pulmonary nodules detected in routine clinical setting.

Methods In two academic centres in the Netherlands, we established a list of patients aged $\geq 40$ years who received a chest CT scan between 2004 and 2012, resulting in 16850 and 23454 eligible subjects. Subsequent diagnosis of lung cancer until the end of 2014 was established through linking with the National Cancer Registry. A nested case-control study was performed (ratio 1:3). Two observers used semiautomated software to annotate the nodules. The Brock model was separately validated on each data set using ROC analysis and compared with a solely sizebased model.

Results After the annotation process the final analysis included 177 malignant and 695 benign nodules for centre $A$, and 264 malignant and 710 benign nodules for centre B. The full Brock model resulted in areas under the curve (AUCs) of 0.90 and 0.91, while the size-only model yielded significantly lower AUCs of 0.88 and 0.87 , respectively $(p<0.001)$. At $10 \%$ malignancy risk, the threshold suggested by the British Thoracic Society, sensitivity of the full model was $75 \%$ and $81 \%$, specificity was $85 \%$ and $84 \%$, positive predictive values were $14 \%$ and $10 \%$ at negative predictive value (NPV) of $99 \%$. The optimal threshold was $6 \%$ for centre $A$ and $8 \%$ for centre B, with NPVs $>99 \%$.

Discussion The Brock model shows high predictive discrimination of potentially malignant and benign nodules when validated in an unselected, heterogeneous clinical population. The high NPV may be used to decrease the number of nodule follow-up examinations.

\section{INTRODUCTION}

Several guidelines provide recommendations on how to manage pulmonary nodules based on the estimated risk of malignancy. ${ }^{1-4}$ Furthermore, several mathematical prediction models have been developed using both clinical and radiological criteria to help clinicians discriminate malignant from benign lesions. ${ }^{5-7}$

McWilliams et $a l^{8}$ published the first risk calculator-known as the Brock or PanCan modelmathematically modelled to the outcome of screen-detected nodules in a large lung cancer screening trial. The model achieved a high

\section{Key messages}

What is the key question?

- Can the Brock model be reliably used as a lung cancer risk calculator in a non-screening population?

What is the bottom line?

- The performance of the Brock lung cancer risk prediction model remains high when applied in an unselected, heterogeneous clinical population.

Why read on?

- The Brock risk calculator has been developed and validated on selected lung cancer screening populations, but has thus far not been tested in a large clinical cohort.

- Our external validation study of a large multicentre clinical population shows persistent discriminative power of the Brock model when used on clinically detected nodules.

performance for predicting malignancy on a per-nodule basis with an area under the curve (AUC) exceeding 0.90. Subsequently, validation of the model using lung cancer screening data from various studies reached similarly high prediction values. ${ }^{9-11}$

In the Lung Imaging Reporting and Data System (Lung-RADS; published by the American College of Radiology), radiologists are encouraged to use the Brock model to decide on the follow-up procedure in CT lung cancer screening. ${ }^{1}$ Similarly, the British Thoracic Society (BTS) released guidelines for the investigation and management of pulmonary nodules in both screening and clinical settings, integrating the Brock model as a risk assessment tool for solitary non-calcified nodules. An empirically set threshold of a 10\% malignancy risk in the Brock model guides the management for each nodule. ${ }^{3}$

Screening participants, in contrary to routine clinical subjects, usually have more small nodules and are subject to specific inclusion and exclusion criteria (eg, heavy smoking and age). Therefore, the high performance of the Brock model in screening cohorts may not automatically be extrapolated to a clinical setting. So far there is only one publication 
evaluating Brock in a clinical context. Al-Ameri et al ${ }^{12}$ reported that the Brock model was superior to other prediction models when applied on clinical data. ${ }^{67}$ However, this study focused on a relatively small population $(n=244)$, excluded pure groundglass nodules and was conducted in a single centre. Thus, the question remains whether the model performs similarly well in a clinical population compared with the screening setting in which it was designed. This study aims to validate the Brock model on an unselected, large and heterogeneous multicentre clinical population.

\section{METHODS}

\section{Study population}

We conducted our study at two academic centres in the Netherlands: University Medical Center Utrecht (centre A) and Radboud University Medical Center, Nijmegen (centre B). In both centres the institutional review board waived the need for informed consent because of its retrospective design and the use of anonymised data.

A list of all subjects aged 40 years and older who received a chest CT scan between January 2004 and December 2012 was established, comprising 16850 subjects from centre A and 23454 from centre B. Subsequently, the list of subjects was cross-referenced to the Netherlands Cancer Registry to acquire information on lung cancer diagnosis until the end of 2014. A total of 1095 and 2124 subjects were diagnosed with lung cancer at centres A and B, respectively. For this study we only included lung cancer cases who had CT imaging available at least 2 months prior to the date of lung cancer diagnosis. By doing so we excluded prevalent lung cancers, for which imaging did not hold any longitudinal information on nodule evolution and future malignancy risk. The interval of 2 months was chosen arbitrarily to balance between longitudinal information and the remaining study group size.

Using the above-mentioned interval, a total of 228 subjects with lung cancer were included from centre $A$ and 405 subjects from centre B. A nested case-control design was applied using a case-control ratio of approximately $1: 3 .{ }^{13}$ 'Cases' were defined as subjects with lung cancer diagnosis, while 'controls' were defined as subjects without the diagnosis of lung cancer during follow-up. Controls were randomly selected from the total cohort of subjects without a lung cancer diagnosis and comprised a total of 684 and 1095 subjects for centres A and B, respectively.

Of all included subjects, both cases and controls, CT scans were retrieved from the Picture Archiving and Communication System. In centre A all scans were reconstructed with $1 \mathrm{~mm}$ thick slices. In centre B the majority of the scans were reconstructed with $3 \mathrm{~mm}$ thick slices. For controls the oldest available CT was collected. For cases all diagnostic CT scans were obtained. Additional exclusions prior to the start of the annotation process were mainly due to retrieval errors and insufficient study quality; in total, an extra 155 cases and 612 controls were excluded. Figure 1 shows the study population selection flow chart.

\section{Annotation process}

Dedicated inhouse software with computer-aided detection (CAD) of both solid and subsolid pulmonary nodules was used for the annotation process (CIRRUS Lung Screening, DIAG, Nijmegen, The Netherlands). All non-calcified nodules $4 \mathrm{~mm}$ or larger were semiautomatically annotated by one observer in each centre (OMM and ETS with 7 and $>20$ years of experience in chest CT). Scans were excluded if subjects were mechanically ventilated, as were scans in which the observer considered a significant part of the lung not assessable (eg, incomplete data, presence of extensive consolidation and severe breathing artefacts).

Observers were instructed to first go through any CAD candidates. True-positive CAD candidates were accepted and false-positive CAD candidates were removed. Subsequently, each scan was visually checked for additional nodules primarily missed by the CAD. Effective nodule diameter and nodule volume were measured semiautomatically using different thresholds for non-solid and solid nodule components. Nodule type (solid, part-solid or non-solid), spiculation (yes/no) and affected lobe were recorded per nodule. In all scans, the presence of emphysema was visually assessed (yes/no).

In the group with lung cancer confirmed by the National Cancer Registry, the date of diagnosis (incidence date) as well as the affected lobe were available. We aimed at identifying each malignancy in the available CT data sets by linking all nodules in the affected lobe across all follow-up scans, assessing nodule morphology and growth pattern. For evaluation in this study, we used the oldest available CT scan on which the lesion was visible. A score of $0-4$ was applied to rate the degree of certainty of the above-mentioned process. A score of 0 referred to no visible lesion in the tumourbearing lobe. A score of 1 referred to a nodule in the tumourbearing lobe without any signs of malignancy (ie, highly unlikely to represent the cancer). A score of 2 referred to an equivocal lesion in the tumour-bearing lobe (ie, no decision possible). A score of 3 referred to a suspicious lesion in the tumour-bearing lobe, but there was no CT scan within 1 month of the incidence date available for definite confirmation (ie, lesion highly likely to represent the lung cancer). A score of 4 referred to a suspicious lesion in the correct lobe and an available CT scan within 1 month of the incidence date (ie, confirmation of the lung cancer). Lesions with a score of 0,1 or 2 were excluded. Hence, only scores of 3 and 4 lesions were included in our analysis, as these were considered to irrefutably represent lung cancers.

In controls, nodules were annotated on the oldest CT scan. All nodules-irrespective of being solitary or multiple per scanwere included in the analysis.

\section{Statistical analysis}

The original Brock publication calculated four models using different sets of input criteria. In the present study the two models that included spiculation as variable were applied to the subjects of centres A and B separately, that is, the full (2b) and parsimonious (1b) Brock model. ${ }^{8}$

According to standard nested case-control analysis, ${ }^{13} 14$ the randomly selected subsets of controls were inflated to simulate a full cohort, resulting in 11770 and 12779 subjects, respectively. Family history was not known in our study population due to the retrospective study design and was set to 0 .

For both validation cohorts discriminative performance of the Brock model was assessed using receiver operating characteristic (ROC) analysis, resulting in AUCs as a performance indicator. A third simple logistic regression model was applied using only nodule size as predictor of lung cancer risk. Differences in discriminative performance were tested between the three models by AUC comparison according to DeLong et al ${ }^{15}$ Sensitivity, specificity, positive predictive value (PPV) and negative predictive value (NPV) were calculated at a threshold of $10 \%$ malignancy risk, the threshold used in the BTS guideline flow chart to select pulmonary nodules for more intense diagnostic work-up. In addition we determined performance at the optimal threshold of the ROC curve (ie, the point on the ROC curve closest to the point where sensitivity and specificity equal 100\%). For comparison, we report 
Center A

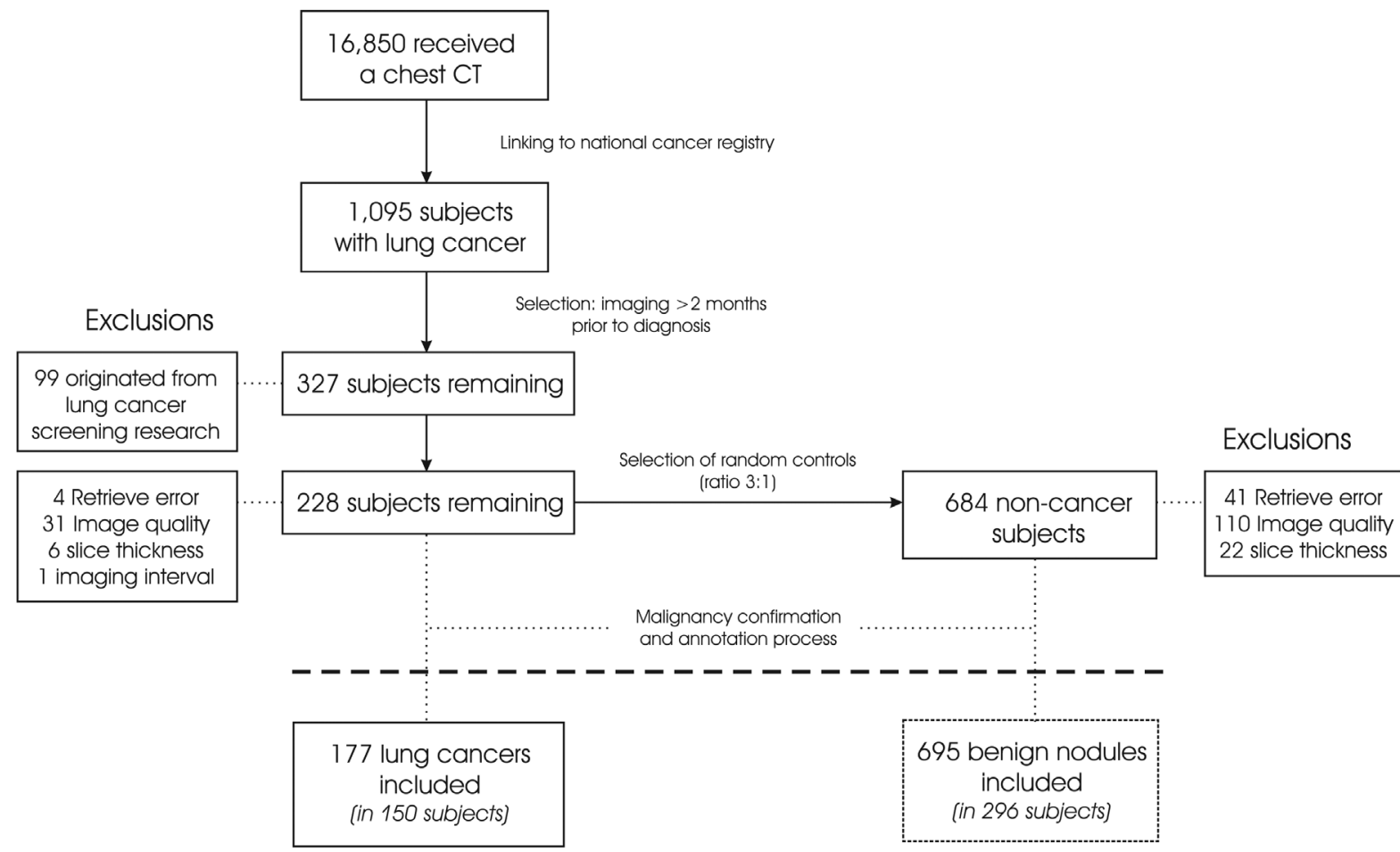

Center B

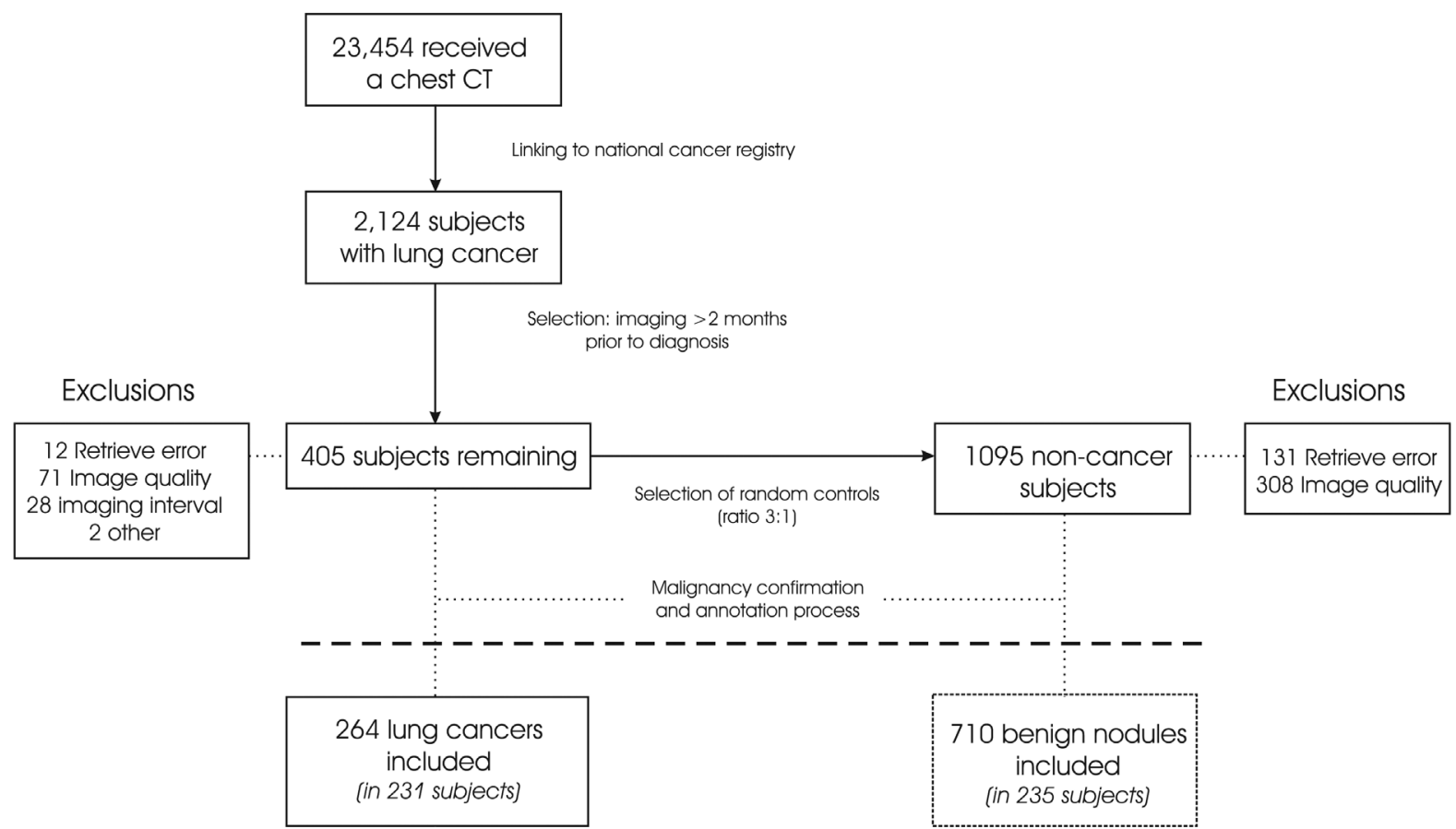

Figure 1 Flow chart of study population inclusion.

performance of the size-only model at the specificity achieved by the full Brock model at a risk threshold of $10 \%$.

\section{RESULTS}

\section{Subject and nodule characteristics}

In total, 441 nodules were given a malignancy score of 3 or 4 (177 in centre A and 264 in centre B). A total of 1405 benign nodules (695 in centre A and 710 in centre B) were found in controls, with a median follow-up of 70 months and 76 months, respectively. For cases, the median time between the annotated $\mathrm{CT}$ and incidence date was 4 months and 2 months for centres $\mathrm{A}$ and $\mathrm{B}$, respectively.

On average, cases were older than controls. The majority of benign and malignant nodules were solid (83\%-88\%). Malignant nodules were larger than benign lesions, were more likely to be located in the upper lobes and were more often spiculated. 


\begin{tabular}{|c|c|c|c|c|}
\hline & \multicolumn{2}{|l|}{ Centre A } & \multicolumn{2}{|l|}{ Centre B } \\
\hline & $\begin{array}{l}\text { Benign } \\
(695)\end{array}$ & $\begin{array}{l}\text { Malignant } \\
\text { (177) }\end{array}$ & $\begin{array}{l}\text { Benign } \\
(710)\end{array}$ & $\begin{array}{l}\text { Malignant } \\
\text { (264) }\end{array}$ \\
\hline Age in years, mean (SD) & $61(12)$ & $65(9)$ & $63(10)$ & $64(9)$ \\
\hline \multicolumn{5}{|l|}{$\operatorname{Sex}(\%)$} \\
\hline Male & $434(62)$ & $89(50)$ & $413(58)$ & $157(59)$ \\
\hline Female & $261(38)$ & $88(50)$ & $297(42)$ & $107(41)$ \\
\hline \multicolumn{5}{|l|}{ Emphysema (\%) } \\
\hline No & $436(63)$ & $61(34)$ & $555(78)$ & $148(56)$ \\
\hline Yes & $259(37)$ & $116(66)$ & $155(22)$ & $116(44)$ \\
\hline \multicolumn{5}{|l|}{ Nodule type (\%) } \\
\hline Non-solid & $84(12)$ & $4(2)$ & $50(7)$ & $11(4)$ \\
\hline Part-solid & $23(3)$ & $26(15)$ & $42(6)$ & $20(8)$ \\
\hline Solid & $588(85)$ & $147(83)$ & $617(87)$ & $233(88)$ \\
\hline \multicolumn{5}{|l|}{ Nodule size (mm) } \\
\hline Median & 5.2 & 17.2 & 8.0 & 20.3 \\
\hline IQR & $4.3-8.6$ & $11.5-27.8$ & $5.9-11.6$ & $13.7-31.2$ \\
\hline \multicolumn{5}{|l|}{ Location (\%) } \\
\hline Lower lobe & $368(53)$ & $60(34)$ & $378(53)$ & $99(37)$ \\
\hline Upper lobe & $327(47)$ & $117(66)$ & $332(47)$ & $165(63)$ \\
\hline \multicolumn{5}{|l|}{ Nodule count } \\
\hline Median & 4 & 3 & 6 & 2 \\
\hline IQR & $2-5$ & $1.5-4$ & $2-11$ & $1-3$ \\
\hline \multicolumn{5}{|l|}{ Spiculation (\%) } \\
\hline No & $694(99.9)$ & $156(88)$ & $702(99)$ & $193(73)$ \\
\hline Yes & $1(0.1)$ & $21(12)$ & $8(1)$ & $71(27)$ \\
\hline
\end{tabular}

IQR from the 25th to the 75th percentile.
In centre $\mathrm{A}$, a higher prevalence of women was seen $(50 \%$ vs $41 \%$, compared with centre B), as well as a higher prevalence of emphysema (66\% vs 44\%). Table 1 lists the study population characteristics for the two centres.

\section{Validation of the Brock model}

The AUCs of the full Brock model were 0.901 and 0.911, respectively. This significantly exceeded the parsimonious model: 0.894 and 0.881 , respectively $(\mathrm{p}<0.05)$ (figures 2 and 3). At a $10 \%$ malignancy risk threshold, sensitivity per centre was $75 \%$ and $81 \%$ (PPV $=10 \%$ and $14 \%)$, and specificity was $86 \%$ and $84 \%(\mathrm{NPV}=99 \%$ and $99 \%)$. The optimal threshold was $6.0 \%$ in centre A (sensitivity of $84 \%$ and specificity of $80 \%$ ) and $8.2 \%$ in centre B (sensitivity of $86 \%$ and specificity of $80 \%$ ).

When using only size to predict the lung cancer risk, an AUC of 0.876 and 0.866 was found for centre A and centre B, respectively. These AUCs were significantly lower $(p<0.001)$ compared with both the full and the parsimonious models (figures 2 and 3 ). Discriminatory performance is summarised in table 2 .

\section{DISCUSSION}

In the past few years, a lot of attention has been paid to optimising the management of screen-detected pulmonary nodules, motivated by the reduction of lung cancer mortality found in the National Lung Screening Trial (NLST). ${ }^{16}$ Especially in a screening setting where the majority of nodules seen on CT are in fact benign, patient management should be personalised using individual nodule risk estimations to save radiation, workload, unnecessary psychological stress and financial expenses.

The same is also true for routine clinical care, because of the increasing use of CT and related increase in the number of incidentally detected pulmonary nodules, and because of the increase in shared decision making by patients and clinicians. ${ }^{17} 18$ Pooling the results of several studies reported a mean nodule

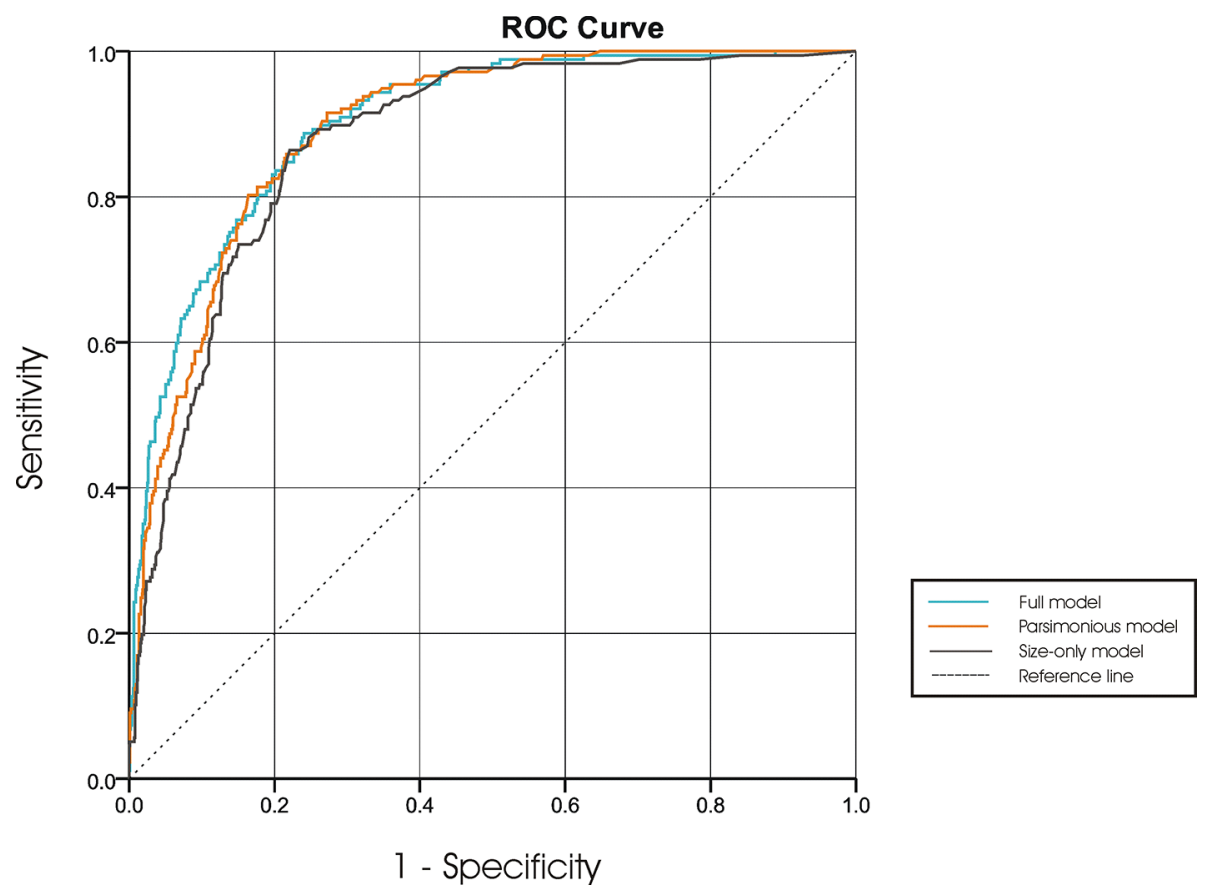

Figure 2 Receiver operating characteristic (ROC) curve of the Brock validation in centre A for the full and parsimonious Brock model ( $p=0.03$ ), as well as the size-only model ( $p<0.001$ compared with both full and parsimonious). 


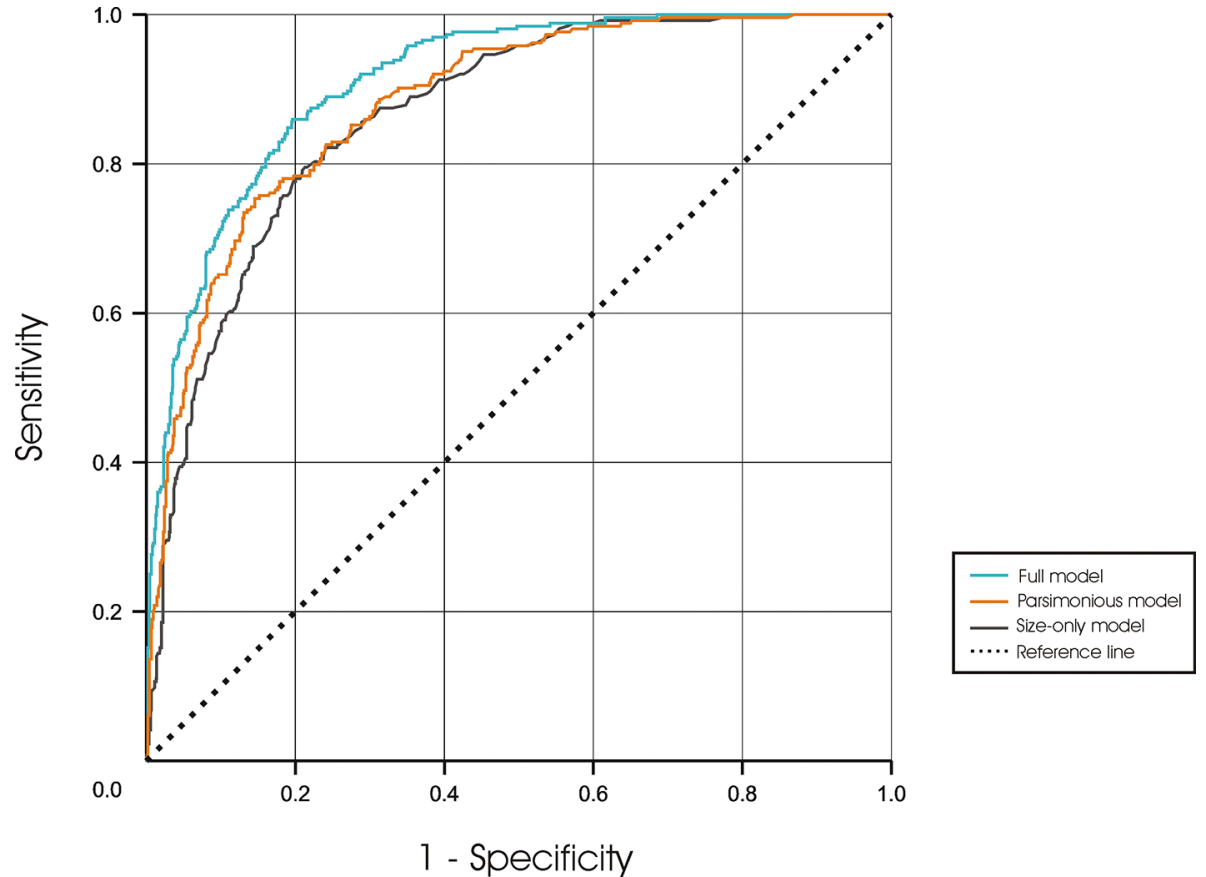

Figure 3 Receiver operating characteristic curve of the Brock validation in centre B for the full and parsimonious Brock model ( $p<0.001)$, as well as the size-only model ( $p<0.001$ compared with both full and parsimonious).

prevalence of $13 \%$ for incidental nodules compared with $33 \%$ for screen-detected nodules with a comparable lung cancer prevalence of $1.5 \%$ and $1.4 \%$, respectively. ${ }^{3}$ Although the reported prevalence of nodules is smaller in a clinical context than in screening, the numbers are substantial and represent a significant body of follow-up CTs for eventually benign nodules.

Mathematically validated risk calculators could be used to help guide the management of pulmonary nodules. The Brock model, developed by McWilliams et $a l,{ }^{8}$ has demonstrated an excellent capacity to discriminate between benign and malignant in screen-detected nodules, and its use is incorporated in nodule management guidelines for both screening and clinical use, ${ }^{13}$ despite validation in clinical subjects was until now only performed once in a small single-centre study. ${ }^{12}$

In this study, we validated the Brock model outside a screening setting, using a large clinical data set from two academic centres. In our unselected and heterogeneous population-even without the restrictions of the Brock model exclusion criteria (eg, previous lung cancer or certain comorbidities) - the model achieved AUCs greater than 0.90 in both data sets. This shows that the malignancy risk calculator is also applicable for incidentally found nodules in daily routine clinical care, although the performance is somewhat lower than in a screening setting.

Table 2 Brock model performance in centres $A$ and $B$

\begin{tabular}{|c|c|c|c|c|c|c|}
\hline & AUC & Risk threshold (\%) & Sensitivity (\%) & Specificity (\%) & PPV (\%) & NPV (\%) \\
\hline \multicolumn{7}{|l|}{ Centre A } \\
\hline \multirow[t]{2}{*}{ Brock (full) } & 0.901 & BTS: 10 & 75 & 86 & 10 & 99 \\
\hline & & Optimum: 6.0 & 84 & 80 & 8 & 100 \\
\hline \multirow[t]{2}{*}{ Brock (parsimonious) } & 0.894 & BTS: 10 & 76 & 85 & 9 & 99 \\
\hline & & Optimum: 9.0 & 80 & 84 & 9 & 100 \\
\hline \multirow[t]{2}{*}{ Size-only } & 0.876 & Equal specificity*: 10.4 & 71 & 86 & 9 & 99 \\
\hline & & Optimum: 4.7 & 86 & 78 & 7 & 100 \\
\hline \multicolumn{7}{|l|}{ Centre B } \\
\hline \multirow[t]{2}{*}{ Brock (full) } & 0.911 & BTS: 10 & 81 & 84 & 14 & 99 \\
\hline & & Optimum: 8.2 & 86 & 80 & 13 & 99 \\
\hline \multirow[t]{2}{*}{ Brock (parsimonious) } & 0.881 & BTS:10 & 80 & 77 & 10 & 99 \\
\hline & & Optimum: 13.8 & 75 & 85 & 15 & 99 \\
\hline \multirow[t]{2}{*}{ Size-only } & 0.866 & Equal specificity*: 3.8 & 71 & 84 & 13 & 99 \\
\hline & & Optimum: 2.8 & 80 & 79 & 11 & 99 \\
\hline
\end{tabular}

* Fixed to equal specificity as the full Brock model at $10 \%$ malignancy risk.

AUC, area under the curve; BTS, British Thoracic Society; NPV, negative predictive value; PPV, positive predictive value. 
Previously, the Brock model was externally validated on screening data from the Danish Lung Cancer Screening Trial and the NLST, showing AUCs ranging between 0.826 and $0.963 .{ }^{9} 10$ White et $a l^{10}$ found the threshold of $10 \%$ malignancy risk to be optimal, with corresponding sensitivity, specificity, PPV and NPV of $85.3 \%, 93.9 \%, 27.4 \%$ and $99.6 \%$, respectively. We found similarly high results for the NPV (99\%), indicating that a risk score $<10 \%$ reliably indicates that a nodule is benign. However, the sensitivities ( $75 \%$ and $81 \%$ vs $85.3 \%$ ), specificities (86\% and $84 \%$ vs $93.3 \%$ ) and PPVs (10\% and $14 \%$ vs $27.4 \%$ ) in our clinical cohorts were inferior, emphasising the different characteristics and challenges of a clinical population compared with a more homogeneous screening population.

Recently, the Brock model was validated on a clinical UK population. ${ }^{12}$ The AUC obtained was 0.90 in a restricted singlecentre cohort $(n=154)$ after applying the same exclusion criteria used in the Brock study. Results were worse $(\mathrm{AUC}=0.868)$ when the model was validated on the full cohort $(n=244)$. In our study, we did not apply any exclusion criteria besides image quality issues and age below 40 years. Moreover, we included all nodule types, while pure ground-glass nodules were excluded in the UK study. ${ }^{12}$

It has to be emphasised that the model achieved similar results in both validation cohorts of our study, despite the CT data being reconstructed with $1 \mathrm{~mm}$ thick slices in centre A and mostly $3 \mathrm{~mm}$ thick slices in centre $\mathrm{B}$, which resulted in different spatial resolution and different accuracy for the assessment of nodule type, spiculation and nodule size.

Although significant, the AUCs show relatively small differences (1\%-5\%), and clinical relevance of these differences therefore has to be interpreted with caution. Still, statistically our study showed that nodule size plays a major role. Although NPV was the same (99\%) when compared with the parsimonious and full Brock models at equal specificity, sensitivity of the size-only model was notably lower. This means that a size-only model is well capable of indicating benign nodules based on a small nodule size, but using only nodule size to predict malignancy shows inferior performance.

Our study has a number of limitations. First, data on lung cancer diagnosis were obtained through the National Cancer Registry, but did not include CT data. We therefore had to use the individual lobe location and incidence date to determine the nodule corresponding to the cancer. Only nodules which were assigned a high level of confidence for representing the known lung cancer were included in the study. This may have introduced a small bias towards more obvious malignancies. Second, for the benign nodules we relied on the outcome data that they did not develop into a malignancy over a median follow-up period of 70 and 76 months in centres $A$ and $B$, respectively. This might have led to some misclassification in non-solid nodules, where development into malignancy might take numerous years. ${ }^{19}$ However, since the National Cancer Registry archives all malignancies irrespective of hospital in the Netherlands and given that the median follow-up period was relatively long, we consider the outcome data reliable. Third, due to the retrospective nature of this study, certain clinical information of the study population was not available, for example, smoking behaviour, symptoms and family history. However, the only parameter that might have had an impact in our study, since it is included in the Brock model, is family history. Nevertheless, since family history did not belong to the group of important input factors in the original Brock model, we consider its influence on our results to be limited. Last, a certain percentage of controls were excluded, mainly because of image quality issues. This may have led to a selection bias. However, given the unselected heterogeneous data set and randomly selected controls, we consider the effect of little importance.

In conclusion, we are the first to validate the Brock risk prediction model in an unselected, large and heterogeneous multicentre clinical population. Our results indicate that the model has a very high negative predictive capacity outside a screening setting. Furthermore, it mirrors the findings from lung cancer screening literature as having a high discriminatory performance, although somewhat inferior. Therefore, we conclude that the Brock risk prediction model is capable of contributing to a personalised risk-dependent lung nodule management outside a screening setting, in an unselected, heterogeneous clinical population. The assumed extrapolation by the guidelines applying the Brock model in clinical subjects seems legitimate.

Acknowledgements The authors thank the registration team of the Netherlands Comprehensive Cancer Organisation (IKNL) for the collection of data for the Netherlands Cancer Registry, as well as the IKNL staff for scientific advice.

Contributors Conception or design of the work: KC, OMM, BvG, MP, PAdJ, CMS-P. Data collection: KC, OMM, PKG, CJ, ETS. Data analysis and interpretation: KC, OMM, AMdH. Drafting the article: KC, OMM, CMS-P. Critical revision of the article: KC, OMM, PKG, CJ, AMdH, ETS, MP, PAdJ, BvG, CMS-P. Final approval of the version to be published: KC, OMM, PKG, CJ, AMdH, ETS, MP, PAdJ, BvG, CMS-P.

Funding This research is funded by a grant from the Dutch Cancer Society (project no KUN 2013-6110).

Competing interests None declared.

Patient consent Not required.

Provenance and peer review Not commissioned; externally peer reviewed.

(c) Article author(s) (or their employer(s) unless otherwise stated in the text of the article) 2018. All rights reserved. No commercial use is permitted unless otherwise expressly granted.

\section{REFERENCES}

1 American College of Radiology. Lung CT Screening Reporting and Data System (LungRADS). http://www.acr.org/Quality-Safety/Resources/LungRADS.

2 National Comprehensive Cancer Network. NCCN Guidelines-Lung Cancer Screening, 2016.

3 Callister ME, Baldwin DR, Akram AR, et al. British Thoracic Society guidelines for the investigation and management of pulmonary nodules. Thorax 2015;70:ii1-ii54.

4 MacMahon H, Naidich DP, Goo JM, et al. Guidelines for management of incidental pulmonary nodules detected on CT Images: from the Fleischner Society 2017. Radiology 2017;284:228-43.

5 Herder GJ, van Tinteren $\mathrm{H}$, Golding RP, et al. Clinical prediction model to characterize pulmonary nodules: validation and added value of $18 \mathrm{~F}$-fluorodeoxyglucose positron emission tomography. Chest 2005;128:2490-6.

6 Gould MK, Ananth L, Barnett PG. A clinical model to estimate the pretest probability of lung cancer in patients with solitary pulmonary nodules. Chest 2007;131:383-8.

7 Swensen SJ, Silverstein MD, Ilstrup DM, et al. The probability of malignancy in solitary pulmonary nodules. Application to small radiologically indeterminate nodules. Arch Intern Med 1997;157:849-55.

8 McWilliams A, Tammemagi MC, Mayo JR, et al. Probability of cancer in pulmonary nodules detected on first screening CT. N Engl J Med 2013;369:910-9.

9 Winkler Wille MM, van Riel SJ, Saghir Z, et al. Predictive Accuracy of the PanCan Lung Cancer Risk Prediction Model -External Validation based on CT from the Danish Lung Cancer Screening Trial. Eur Radiol 2015;25:3093-9.

10 White CS, Dharaiya E, Campbell E, et al. The Vancouver Lung Cancer Risk Prediction Model: Assessment by Using a Subset of the National Lung Screening Trial Cohort. Radiology 2017;283.

11 Zhao H, Marshall HM, Yang IA, et al. Screen-detected subsolid pulmonary nodules: long-term follow-up and application of the PanCan lung cancer risk prediction model. Br J Radiol 2016;89:.

12 Al-Ameri A, Malhotra P, Thygesen $\mathrm{H}$, et al. Risk of malignancy in pulmonary nodules: A validation study of four prediction models. Lung Cancer 2015;89:27-30.

13 Biesheuvel CJ, Vergouwe Y, Oudega R, et al. Advantages of the nested case-control design in diagnostic research. BMC Med Res Methodol 2008;8:48.

14 Ernster VL, studies Ncase-controlPrev. Med 1994;23:587-90.

15 DeLong ER, DeLong DM, Clarke-Pearson DL. Comparing the areas under two or more correlated receiver operating characteristic curves: a nonparametric approach. Biometrics 1988;44:837-45. 
16 Aberle DR, Adams AM, Berg CD, et al. Reduced lung-cancer mortality with low-dose computed tomographic screening. N Engl J Med 2011;365:395-409.

17 Callister ME, Baldwin DR. How should pulmonary nodules be optimally investigated and managed? Lung Cancer 2016;91:48-55.
18 Pedersen JH, Saghir Z, Wille MM, et al. Ground-Glass opacity lung nodules in the era of lung cancer CT screening: radiology, pathology, and clinical management. Oncology 2016;30:266-74.

19 Scholten ET, de Jong PA, de Hoop B, et al. Towards a close computed tomography monitoring approach for screen detected subsolid pulmonary nodules? Eur Respir $J$ 2015:45:765-73. 